\title{
ON ROOTED PROPERTIES OF COMPLEX ANALYTIC SYSTEMS*
}

\author{
by XIAO-QIANG ZHAO
}

(Received 24th July 1992)

\begin{abstract}
This paper is devoted to the study of rooted properties of phase surfaces defined by complex analytic systems. We first obtain the Rooted Theorem of Analytic Systems. Then we prove the Generalized Strong Rooted Theorem of $\left(E_{n}^{m}\right)(m \geqq 2)$, which implying the Strong Rooted Theorem of a Class of $\left(E_{n}^{3}\right)$.
\end{abstract}

1991 Mathematics subject classification: 34A20, 34C35, 58F25.

\section{Rooted theorem of analytic systems}

Consider differential equations in complex domain

$$
\frac{d W}{d T}=F(W) \quad W \in C^{m}
$$

where $C^{m}$ denotes the $m$-dimensional complex spaces, and $F: C^{m} \rightarrow C^{m}$ is an analytic function. By Cauchy's theorem, equation (1.1) has a unique local analytic solution satisfying $T=0, W=W_{0}$, denoting $W=W\left(T, W_{0}\right),|T|<r(r>0)$. Taking $W\left(T, W_{0}\right)$ as an element of analytic function, we obtain a global analytic function $\Phi\left(T, W_{0}\right)$ with $D\left(W_{0}\right)$ as its domain of definition. It can be proved that $D\left(W_{0}\right)$ is an open subset of $C^{1}$. If $\Phi\left(T, W_{0}\right)$ is analytically continuable along arcline $L$, we denote it by $\Phi^{L}\left(T, W_{0}\right)$.

Definition 1. The set

$$
\Sigma\left(W_{0}\right)=\left\{W ; W=\Phi\left(T, W_{0}\right), T \in D\left(W_{0}\right)\right\}
$$

is called the phase surface of (1.1) through $W_{0} \in C^{m}$.

Obviously, if $W_{0}$ is a finite singular point of (1.1), i.e. $F\left(W_{0}\right)=0$, then $\Phi\left(T, W_{0}\right) \equiv W_{0}$, $T \in C^{1}$, and hence $\Sigma\left(W_{0}\right)=\left\{W_{0}\right\}$.

Proposition 1. Let $\Sigma$ be a phase surface of (1.1). If $W_{0} \in \bar{\Sigma}$, then $\Sigma \supset \Sigma\left(W_{0}\right)$.

Proof. For the simplicity, let $\Sigma_{0}=\Sigma\left(W_{0}\right)$ and $D_{0}=D\left(W_{0}\right)$. For any $W_{1} \in \Sigma_{0}$, there

* Research supported by the National Science Foundation of P.R. China. 
exist $T_{1} \in D_{0}$ and simple curve $L \subset D_{0}$ connecting 0 and $T_{1}$ such that $W_{1}=\Phi^{L}\left(T_{1}, W_{0}\right)$. By the continuous dependence theorem of solutions on initial values [5], there exists a neighbourhood $U_{0}$ of $W_{0}$, such that

$$
\lim _{\substack{W \rightarrow W_{0} \\ W \in U_{0}}} \Phi^{L}(T, W)=\Phi^{L}\left(T, W_{0}\right)
$$

uniformly for $T \in L$. As $W_{0} \in \bar{\Sigma}$, there exist $W_{n} \in \Sigma(n=1,2, \ldots)$ such that $\lim _{n \rightarrow \infty} W_{n}=W_{0}$. Then there exists $N>0$ such that when $n \geqq N, W_{n} \in U_{0}, L \subset D\left(W_{n}\right)$ and

$$
\lim _{n \rightarrow \infty} \Phi^{L}\left(T, W_{n}\right)=\Phi^{L}\left(T, W_{0}\right)
$$

uniformly for $T \in L$. Especially,

$$
\lim _{n \rightarrow \infty} \Phi^{L}\left(T_{1}, W_{n}\right)=\Phi^{L}\left(T_{1}, W_{0}\right)=W_{1}
$$

As $W_{n} \in \Sigma$, then $\Phi^{L}\left(T_{1}, W_{n}\right) \in \Sigma(n \geqq N)$. Therefore $W_{1} \in \bar{\Sigma}$. So $\bar{\Sigma} \supset \Sigma_{0}$, and hence $\bar{\Sigma} \supset \bar{\Sigma}_{0}$. This completes the proof.

Proposition 2. Consider equation (1.1) with $m=1$ and let $C_{0}=\{W ; F(W)=0\}$. Then for any $W_{0} \in C^{1} \backslash C_{0}, \Sigma\left(W_{0}\right)=C^{1} \backslash C_{0}$.

Proof. For any given $W_{1} \in C^{1} \backslash C_{0}$, choose a simply-connected domain such that $W_{0} \in G, W_{1} \in G$, and $C_{0} \cap G=\varnothing(\varnothing$ being empty set). So for any $W \in G$, we can define

$$
H(W)=\int_{W_{0}}^{W} \frac{d W}{F(W)} .
$$

Then, as $d T / d W=1 / F(W), H(W)=T-T_{0}$ is an integral of (1.1) and satisfies $W=W_{0}$, $T=T_{0}$. Therefore, $G \subset \Sigma\left(W_{0}\right)$, and hence $W_{1} \in \Sigma\left(W_{0}\right)$. Then $\Sigma\left(W_{0}\right)=C^{1} \backslash C_{0}$.

It is easy to prove the following:

Lemma 1. Suppose that the sequence of points $\left(w_{1}^{(n)}, \ldots, w_{m}^{(n)}\right) \in C^{m}$ satisfies

$$
\lim _{n \rightarrow \infty}\left(\left|w_{1}^{(n)}\right|+\cdots+\left|w_{m}^{(n)}\right|\right)=+\infty
$$

then there must exist a subsequence $\left(w_{1}^{\left(n_{k}\right)}, \ldots, w_{m}^{\left(n_{k}\right)}\right)(k=1,2, \ldots)$ such that $\lim _{k \rightarrow \infty}\left|w_{i}^{\left(n_{k}\right)}\right|=$ $+\infty$ for some $i(1 \leqq i \leqq m)$ and $w_{j}^{\left(n_{k}\right)} / w_{i}^{\left(n_{k}\right)}(k=1,2, \ldots)$ converges for all $j(1 \leqq j \leqq m, j \neq i)$.

Definition 2. Let $C P^{m}$ denote the $m$-dimensional complex projective space. A given phase surface $\Sigma$ of $(1.1)$ is said to have the point at infinity $\left(W_{1}^{0}, \ldots, W_{m}^{0}, 0\right) \in C P^{m}$ as its 
limit point if there exists a sequence of points $\left(w_{1}^{(n)}, \ldots, w_{m}^{(n)}\right) \in \Sigma(n=1,2, \ldots, \infty)$ such that for some $i(1 \leqq i \leqq m)$

$$
W_{i}^{0} \neq 0, \quad \lim _{n \rightarrow \infty}\left|w_{i}^{(n)}\right|=+\infty
$$

and for all $j(1 \leqq j \leqq m, j \neq i)$

$$
\lim _{n \rightarrow \infty} \frac{w_{j}^{(n)}}{w_{i}^{(n)}}=\frac{W_{j}^{0}}{W_{i}^{0}}
$$

Theorem 1. For any given point $W_{0} \in C^{m}$ which is not a finite singular point of (1.1), the phase surface $\Sigma\left(W_{0}\right)$ has some point at infinity as its limit point.

Proof. First we prove that $\Sigma\left(W_{0}\right)$ is unbounded. Suppose $\Sigma\left(W_{0}\right)$ is bounded, then we can infer that $\partial D\left(W_{0}\right)=\varnothing$. Assume it is not so, then there exist $T^{*} \in \partial D\left(W_{0}\right)$ and a simple curve $L$ connecting 0 and $T^{*}$ such that $L_{1}=L-T^{*} \subset D\left(W_{0}\right)$ and $\Phi\left(T, W_{0}\right)$ is analytically continuable along $L_{1}$. Choose a sequence $T_{n} \in L_{1}$ such that $T_{n} \rightarrow T^{*}(n \rightarrow \infty)$. As the sequence of points $W_{n}=\Phi^{L_{1}}\left(T_{n}, W_{0}\right) \in \Sigma\left(W_{0}\right)$ is bounded, without loss of generality, we can assume $W_{n} \rightarrow W^{*}(n \rightarrow \infty), W^{*} \in C^{m}$. By the continuation theorem of solutions [5], $T^{*} \in D\left(W_{0}\right)$. This is contrary to the fact that $T^{*} \in \partial D\left(W_{0}\right)$ and $D\left(W_{0}\right)$ is an open subset of $C^{1}$. So $D\left(W_{0}\right)=C^{1}$. As $C^{1}$ is simply connected region, by Riemann's monodromy theorem [1], $\Phi\left(T, W_{0}\right)$ is a bounded global function. By Liouville's theorem, $\Phi\left(T, W_{0}\right) \equiv$ Const. So $W_{0}$ is a finite singular point of (1.1). This is contrary to our assumption for $W_{0}$.

As $\Sigma\left(W_{0}\right)$ is unbounded, by Lemma 1 given above, there exists a sequence $\left(w_{1}^{(n)}, \ldots, w_{m}^{(n)}\right) \in \Sigma\left(W_{0}\right)$ such that for some $i(1 \leqq i \leqq m)$

$$
\lim _{n \rightarrow \infty}\left|w_{i}^{(n)}\right|=+\infty
$$

and for all $j(1 \leqq j \leqq m, j \neq i), w_{j}^{(n)} / w_{i}^{(n)}$ converges, saying, to $W_{j}^{0} \in C^{1}$. So, by Definition 2 , $\Sigma\left(W_{0}\right)$ has the point at infinity $\left(W_{1}^{0}, \ldots, W_{i-1}^{0}, 1, W_{i+1}^{0}, \ldots, W_{m}^{0}, 0\right)$ as its limit point. This completes our proof.

Note 1. For the two dimensional complex polynomical systems, by using the theory of real equation, Y.S. Chin proved the "Rooted Theorem" [2]:

Each solution surface either has some finite singular point as its limit point or extends to infinity.

It is easy to see that Theorem 1 for $m=2$ is a strong form of Chin's.

Example 1. For the analytic system

$$
\left\{\begin{array}{l}
\frac{d w}{d T}=w \cos x \\
\frac{d z}{d T}=1
\end{array}\right.
$$


any phase surface has the point at infinity $(0,1,0) \in P^{2} C$ as its limit point. In fact, this system has the general solution

$$
w=c e^{\sin z}
$$

For any given $c$, the corresponding phase surface

$$
\Sigma_{c}=\left\{\left(c e^{\sin z}, z\right) ; z \in C^{1}\right\}
$$

Choose a sequence of points $\left(w_{n}, z_{n}\right)=\left(c e^{\sin n}, n\right) \in \Sigma_{c}$, then $\left|z_{n}\right|=n \rightarrow \infty$, and

$$
\frac{w_{n}}{z_{n}}=\frac{c e^{\sin n}}{n} \rightarrow 0(n \rightarrow \infty)
$$

\section{Generalized strong rooted theorem of $\left(E_{n}^{m}\right)(m \geqq 2)$}

Let the sign "deg" denote the degree of polynomial. Consider the polynomial systems:

$$
\frac{d w_{i}}{d T}=P_{i}\left(w_{1}, w_{2}, \ldots, w_{m}\right)(i=1,2, \ldots, m)\left(E_{n}^{m}\right)
$$

where $P_{i}\left(w_{1}, w_{2}, \ldots, w_{m}\right)$ is $m$-variable polynomial $(i=1,2, \ldots, m)$

$$
\max _{1 \leqq i \leqq m}\left\{\operatorname{deg} P_{i}\left(w_{1}, w_{2}, \ldots, w_{m}\right)\right\}=n,
$$

and $P_{1}\left(w_{1}, w_{2}, \ldots, w_{m}\right), \ldots, P_{m}\left(w_{1}, w_{2}, \ldots, w_{m}\right)$ have no common factors. Let $P_{i}^{*}\left(w_{1}, \ldots, w_{m}\right)$ be the $n$-degree homogeneous polynomial of $P_{i}\left(w_{1}, \ldots, w_{m}\right)$ and denote

$$
\bar{P}_{i}\left(W_{1}, W_{2}, \ldots, W_{m}, M\right)=M^{n} P_{i}\left(\frac{W_{1}}{M}, \frac{W_{2}}{M}, \ldots, \frac{W_{m}}{M}\right)(i=1,2, \ldots, m)
$$

Then $P_{i}^{*}\left(W_{1}, W_{2}, \ldots, W_{m}\right)=\bar{P}_{i}\left(W_{1}, W_{2}, \ldots, W_{m}, 0\right)$.

Taking the transformation $T_{i}:(i=1,2, \ldots, m)$

$$
w_{1}=\frac{W_{1}}{M}, \ldots, w_{i-1}=\frac{W_{i-1}}{M}, w_{i}=\frac{1}{M}, w_{i+1}=\frac{W_{i+1}}{M}, \ldots, w_{m}=\frac{W_{m}}{M}
$$

then $\left(E_{n}^{m}\right)$ is transformed into 


$$
\left\{\begin{aligned}
\frac{d W_{j}}{d T^{\prime}}= & W_{j} \bar{P}_{i}\left(W_{1}, \ldots, W_{i-1}, 1, W_{i+1}, \ldots, W_{m}, M\right) \\
& -\bar{P}_{j}\left(W_{1}, \ldots, W_{i-1}, 1, W_{i+1}, \ldots, W_{m}, M\right)(j=1,2, \ldots, m, j \neq i) \\
\frac{d M}{d T^{\prime}}= & M \bar{P}_{i}\left(W_{1}, \ldots, W_{i-1}, 1, W_{i+1}, \ldots, W_{m}, M\right) .
\end{aligned}\right.
$$

Make a new $(m-1)$-dimensional polynomial system:

$$
\begin{aligned}
\frac{d W_{j}}{d T^{\prime}}= & W_{j} P_{i}^{*}\left(W_{1}, \ldots, W_{i-1}, 1, W_{i+1}, \ldots, W_{m}\right) \\
& -P_{j}^{*}\left(W_{1}, \ldots, W_{i-1}, 1, W_{i+1}, \ldots, W_{m}\right)(j=1,2, \ldots, m, j \neq i)
\end{aligned}
$$

and let

$$
\begin{gathered}
I S_{i}=\left\{\left(W_{1}, \ldots, W_{i-1}, 1, W_{i+1}, \ldots, W_{m}, 0\right) \in C P^{m}\right. \\
\left.\left(W_{1}, \ldots, W_{i-1}, W_{i+1}, \ldots, W_{m}\right) \text { is a finite singular point of }(2.2)\right\}
\end{gathered}
$$

Definition 3. The set $I S=\bigcup_{i=1}^{m} I S_{i}$ is called the set of singular points at infinity of $\left(E_{n}^{m}\right)$.

Definition 4. By induction, we define the set of generalized singular points at infinity of $\left(E_{n}^{m}\right)$, denoting it by GIS.

(1) For $m=2$, define $G I S=I S$.

(2) Assume GIS for $(m-1)$ dimensional polynomial systems has been defined, then let $I S^{\prime}=\bigcup_{i=1}^{m} I S_{i}^{\prime}$, where

$$
I S_{i}^{\prime}=\left\{\left(W_{1}, \ldots, W_{i-1}, 0, W_{i+1}, \ldots, W_{m}, 0\right) \in C P^{m}\right.
$$

$\left(W_{1}, \ldots, W_{i-1}, W_{i+1}, \ldots, W_{m}, 0\right)$ is a generalized singular point at infinity of (2.2)

and define $G I S=I S \cup I S^{\prime}$.

Theorem 2. For any given point $W_{0} \in C^{m}$ which is not a finite singular point of $\left(E_{n}^{m}\right)$, the phase surface $\Sigma\left(W_{0}\right)$ has some generalized singular point at infinity as its limit point.

Proof. By mathematical induction.

(1) For $m=2$, by Theorem 1 in Section 1, without loss of generality, assume $\Sigma=\Sigma\left(W_{0}\right)$ contains the points at infinity $\left(1, W_{2}^{0}, 0\right) \in C P^{2}$. Take the transformation $T_{1}$ :

$$
w_{1}=\frac{1}{M} \quad w_{2}=\frac{W_{2}}{M}
$$

then $\left(E_{n}^{2}\right)$ is transformed into 


$$
\left\{\begin{array}{l}
\frac{d W_{2}}{d T^{\prime}}=W_{2} \bar{P}_{1}\left(1, W_{2}, M\right)-\bar{P}_{2}\left(1, W_{2}, M\right) \\
\frac{d M}{d T^{\prime}}=M \bar{P}_{1}\left(1, W_{2}, M\right)
\end{array}\right.
$$

with a phase surface $\Sigma_{1}$ corresponding to $\Sigma$. As $\Sigma$ contains $\left(1, W_{2}^{0}, 0\right) \in P^{2}$, then $\left(W_{2}^{0}, 0\right) \in \bar{\Sigma}_{1}$. There are two cases:

(i) If $\left(W_{2}^{0}, 0\right)$ is a finite singular point of (2.3), then, by the definition of $I S_{1}$, $\left(1, W_{2}^{0}, 0\right) \in I S_{1}$. Therefore the conclusion holds.

(ii) If $\left(W_{2}^{0}, 0\right)$ is not any finite singular point of $(2.3)$, then

$$
W_{2}^{0} P_{1}^{*}\left(1, W_{2}^{0}\right)-P_{2}^{*}\left(1, W_{2}^{0}\right) \neq 0 \text {. }
$$

Let $\Sigma_{0}$ be the phase surface of $(2.3)$ through $\left(W_{2}^{0}, 0\right)$ then

$$
\Sigma_{0}=\left\{\left(W_{2}, 0\right) ; W_{2} \in \Sigma_{0}^{\prime}\right\}
$$

where $\Sigma_{0}^{\prime}$ is the phase surface through $W_{2}^{0} \in C^{1}$ of the following equation

$$
\frac{d W_{2}}{d T^{\prime}}=W_{2} P_{1}^{*}\left(1, W_{2}\right)-P_{2}^{*}\left(1, W_{2}\right)
$$

As $W_{2}^{0} P_{1}^{*}\left(1, W_{2}^{0}\right)-P_{2}^{*}\left(1, W_{2}^{0}\right) \neq 0$, by Proposition 2 in Section 1 ,

$$
\Sigma_{0}^{\prime}=C^{1} \backslash C_{0}
$$

where $C_{0}=\left\{W_{2} ; W_{2} P^{*}\left(1, W_{2}\right)-P_{2}^{*}\left(1, W_{2}\right)=0\right\}$. Then

$$
\Sigma_{0}=\left\{\left(W_{2}, 0\right) ; W_{2} \in C^{1} \backslash C_{0}\right\}
$$

Since $\left(W_{2}^{0}, 0\right) \in \bar{\Sigma}_{1}$, by Proposition 1 in Section 1 ,

$$
\bar{\Sigma}_{1} \supset \bar{\Sigma}_{0}=\left\{\left(W_{2}, 0\right) ; W_{2} \in C^{1}\right\}
$$

Then for any $W_{2} \in C^{1}, \Sigma$ has the point at infinity $\left(1, W_{2}, 0\right) \in C P^{2}$ as its limit point.

On the other hand, as $\Sigma_{0}$ is unbounded, there exists a sequence of points $\left(W_{2}^{\prime(n)}, 0\right) \in \Sigma_{0}$ such that

$$
\left.\mid W_{2}^{\prime(n)}\right) \rightarrow+\infty(n \rightarrow \infty)
$$

Since $\Sigma_{1} \supset \bar{\Sigma}_{0}$, there exists a sequence of points $\left(W^{(n)}, M^{(n)}\right) \in \Sigma_{1}$ such that 


$$
\left|W_{2}^{(n)}-W_{2}^{(n)}\right|<\frac{1}{n},\left|M^{(n)}\right|<\frac{1}{n}(n=1,2, \ldots)
$$

Then

$$
\left|W_{2}^{(n)}\right| \rightarrow+\infty, M^{(n)} \rightarrow O(n \rightarrow \infty) .
$$

Therefore there exists a sequence of points $\left(w_{1}^{(n)}, w_{2}^{(n)}\right) \in \Sigma$ correspondingly such that

$$
\left|w_{2}^{(n)}\right|=\frac{\left|W_{2}^{(n)}\right|}{\left|M^{(n)}\right|} \rightarrow+\infty, \frac{\left|w_{1}^{(n)}\right|}{\left|w_{2}^{(n)}\right|}=\frac{1}{W_{2}^{(n)}} \rightarrow 0,(n \rightarrow \infty) .
$$

Then $\Sigma$ has the point $(0,1,0) \in C P^{2}$ as its limit point.

So $\Sigma$ has all points at infinity in $C P^{2}$ and hence all singular points at infinity as its limit point.

From (i) and (ii), the conclusion of Theorem 2 for $m=2$ holds.

(2) Suppose that Theorem 2 for $(m-1)$ holds. By Theorem 1 in Section 1 , without loss of generality, assume $\Sigma=\Sigma\left(W_{0}\right)$ has the point at infinity $\left(1, W_{2}^{0}, \ldots, W_{m}^{0}, 0\right) \in C P^{m}$ as its limit point. Take the transformation $T_{1}$ :

$$
w_{1}=\frac{1}{M}, w_{2}=\frac{W_{2}}{M}, \ldots, w_{m}=\frac{W_{m}}{M}
$$

then $\left(E_{n}^{m}\right)$ is transformed into

$$
\left\{\begin{array}{l}
\frac{d W_{j}}{d T^{\prime}}=W_{j} \bar{P}_{1}\left(1, W_{2}, \ldots, W_{m}, M\right)-\bar{P}_{j}\left(1, W_{2}, \ldots, W_{m}, M\right)(2 \leqq j \leqq m) \\
\frac{d M}{d T^{\prime}}=M \bar{P}_{1}\left(1, W_{2}, \ldots, W_{m}, M\right)
\end{array}\right.
$$

and $\Sigma$ corresponds to $\Sigma_{1}$. As $\Sigma$ has $\left(1, W_{2}^{0}, \ldots, W_{m}^{0}, 0\right) \in C P^{m}$ as its limit point, then $\left(W_{2}^{0}, \ldots, W_{m}^{0}, 0\right) \in \Sigma_{1}$. There are two cases:

(i) If $\left(W_{2}^{0}, \ldots, W_{m}^{0}, 0\right)$ is a finite singular point of $(2.5)$, then, by the definition of $I S_{1}$, $\left(1, W_{2}^{0}, \ldots, W_{m}^{0}, 0\right) \in I S_{1}$.

(ii) If $\left(W_{2}^{0}, \ldots, W_{m}^{0}, 0\right)$ is not any finite singular point of $(2.5)$, then $\left(W_{2}^{0}, \ldots, W_{m}^{0}\right)$ is not any finite singular point of the following equation

$$
\frac{d W_{j}}{d T^{\prime}}=W_{j} P_{1}^{*}\left(1, W_{2}, \ldots, W_{m}\right)-P_{j}^{*}\left(1, W_{2}, \ldots, W_{m}\right),(2 \leqq j \leqq m)
$$

Let $\Sigma_{0}$ be the phase surface of $(2.5)$ through $\left(W_{2}^{0}, \ldots, W_{m}^{0}, 0\right)$, then

$$
\Sigma_{0}=\left\{\left(W_{2}, \ldots, W_{m}, 0\right) ;\left(W_{2}, \ldots, W_{m}\right) \in \Sigma_{0}^{\prime}\right\}
$$

where $\Sigma_{0}^{\prime}$ is the phase surface of $(2.6)$ through $\left(W_{2}^{0}, \ldots, W_{m}^{0}\right)$. As $(2.6)$ is a $(m-1)$ - 
dimensional polynomial system, by our assumption, $\Sigma_{0}^{\prime}$ has some generalized singular point at infinity $\left(\bar{W}_{2}^{0}, \bar{W}_{3}^{0}, \ldots, \bar{W}_{m}^{0}, 0\right) \in C P^{m-1}$ of $(2: 6)$ as its limit point. Without loss of generality, assume that $\bar{W}_{2}^{0} \neq 0$ and there exists a sequence of points $\left(W_{2}^{\prime(n)}, \ldots, W_{m}^{\prime(n)}\right) \in \Sigma_{0}^{\prime}$ such that

$$
\left|W_{2}^{\prime(n)}\right| \rightarrow+\infty, \frac{W_{j}^{\prime(n)}}{W_{2}^{(n)}} \rightarrow \frac{\bar{W}_{j}^{0}}{\bar{W}_{2}^{0}}(3 \leqq j \leqq m),(n \rightarrow \infty)
$$

As $\left(W_{2}^{0}, \ldots, W_{m}^{0}, 0\right) \in \bar{\Sigma}_{1}$, by Proposition 1 in Section $1, \bar{\Sigma}_{1} \supset \Sigma_{0}$. Therefore for any given $n,(n=1,2, \ldots, \infty)$, since $\left(W_{2}^{\prime(n)}, \ldots, W_{m}^{(n)}, 0\right) \in \Sigma_{0}$, there exists $\left(W_{2}^{(n)}, \ldots, W_{m}^{(n)}, M^{(n)}\right) \in \Sigma_{1}$ such that

$$
\left|W_{j}^{(n)}-W_{j}^{(n)}\right|<\frac{1}{n}(2 \leqq j \leqq m),\left|M^{(n)}\right|<\frac{1}{n}
$$

Then $\left|W_{2}^{(n)}\right| \rightarrow+\infty(n \rightarrow \infty)$ and

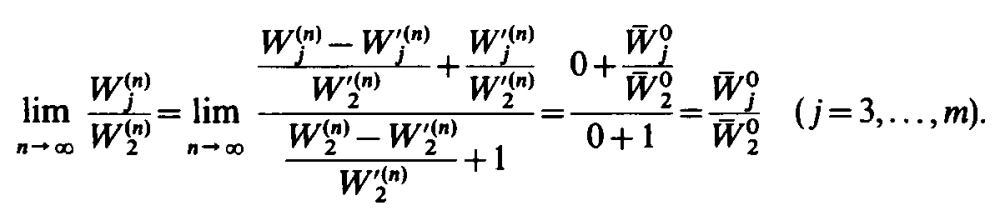

Therefore there exists a sequence of points $\left(w_{1}^{(n)}, \ldots, w_{m}^{(n)}\right) \in \Sigma$ correspondingly such that

and

$$
\left|w_{2}^{(n)}\right|=\frac{\left|W_{2}^{(n)}\right|}{\left|M^{(n)}\right|} \rightarrow+\infty, \frac{w_{1}^{(n)}}{w_{2}^{(n)}}=\frac{1}{W_{2}^{(n)}} \rightarrow O(n \rightarrow \infty)
$$

$$
\frac{w_{j}^{(n)}}{w_{2}^{(n)}}=\frac{W_{j}^{(n)}}{W_{2}^{n}} \rightarrow \frac{\bar{W}_{j}^{0}}{\bar{W}_{2}^{0}}(3 \leqq j \leqq m)(n \rightarrow \infty)
$$

Then $\Sigma$ has the point at infinity $\left(0, \bar{W}_{2}^{0}, \ldots, \bar{W}_{m}^{0}, 0\right) \in C P^{m}$ as its limit point. By the definition of $I S_{1}^{\prime},\left(0, \bar{W}_{2}^{0}, \ldots, \bar{W}_{m}^{0}, 0\right) \in I S_{1}^{\prime}$.

From (i) and (ii), the conclusion of Theorem 2 for $m$ holds.

By mathematical induction, Theorem 2 for all $m \geqq 2$ is true. This completes the proof.

From (2) in the process of the proof above, we can obtain the following:

Lemma 2. Let $\Sigma$ be a phase surface of $\left(E_{n}^{m}\right)$ which is not a finite singular point of it. If $\Sigma$ has the point at infinity $\left(W_{1}^{0}, W_{2}^{0}, \ldots, W_{m}^{0}, 0\right) \in C P^{m}$ as its limit point and $W_{i}^{0} \neq 0$ for some $i(1 \leqq i \leqq m)$, then it must have some point at infinity in $I S_{i} \cup I S_{i}^{\prime}$ as its limit point.

Note 2. Let $\Sigma$ be a phase surface of $\left(E_{n}^{m}\right)$. If $\Sigma$ has some finite singular point or 
singular point at infinity $P$ of $\left(E_{n}^{m}\right)$ as its limit point, we call $P$ as a "root" of $\Sigma$. For $m=2$, Y. S. Chin proved the "Strong Rooted Theorem" [4]:

Each solution surface of $\left(E_{n}^{2}\right)$ has some finite singular point or singular point at infinity as its root.

As $G I S=I S$ for $\left(E_{n}^{2}\right)$, it is easy to see that Theorem 2 for $m=2$ is a strong form of Chin's.

Note 3. For $\left(E_{n}^{m}\right)$ in real domain, there is no parallel result to Theorem 2. This can be seen from the following two simple counterexamples.

Example 2. For the two-dimensional system

$$
\left\{\begin{array}{l}
\frac{d w}{d T}=-z \\
\frac{d z}{d T}=w
\end{array}\right.
$$

by concrete calculation, $I S=\{(1, \pm i, 0)\}$. This system has the general phase surfaces

Let

$$
\Sigma_{c}: w^{2}+z^{2}=c .
$$

$$
w=\frac{W}{M} \quad z=\frac{Z}{M}
$$

$\Sigma_{c}$ is extended into $\Sigma_{c}^{\prime}$ in $C P^{2}$

$$
\Sigma_{c}^{\prime}: W^{2}+Z^{2}-c M^{2}=0
$$

It is easy to verify that $\Sigma_{c}$ has both singular points at infinity $(1, \pm i, 0) \in C P^{2}$ as its limit points.

Example 3. For the three-dimensional system

$$
\left\{\begin{array}{l}
\frac{d w_{1}}{d T}=w_{3}-w_{2} \\
\frac{d w_{2}}{d T}=w_{1}-w_{3} \\
\frac{d w_{3}}{d T}=w_{2}-w_{1}
\end{array}\right.
$$


by concrete calculation, $I S_{1}=I S_{2}=I S_{3}=\{(1,1,1,0),(1,(-1 \pm \sqrt{3} i) / 2,(-1 \mp \sqrt{3} i) / 2,0)\}$. This system has general phase surfaces

Let

$$
\Sigma: \begin{aligned}
& w_{1}^{2}+w_{2}^{2}+w_{3}^{2}=c_{1} \\
& w_{1}+w_{2}+w_{3}=c_{2}
\end{aligned}
$$

$$
w_{1}=\frac{W_{1}}{M} \quad w_{2}=\frac{W_{2}}{M} \quad w_{3}=\frac{W_{3}}{M}
$$

then $\Sigma$ is extended into $\Sigma^{\prime}$ in $C P^{3}$

$$
\Sigma^{\prime}: \begin{aligned}
& W_{1}^{2}+W_{2}^{2}+W_{3}^{2}-c_{1} M^{2}=0 \\
& W_{1}+W_{2}+W_{3}-c_{2} M=0
\end{aligned}
$$

It is easy to verify that $\Sigma$ has two singular points at infinity $(1,(-1 \pm \sqrt{3} i) / 2,(-1 \mp \sqrt{3} i) / 2,0) \in C P^{3}$ as its limit points.

\section{Strong rooted theorem of a class of $\left(E_{n}^{3}\right)$}

In this section, on the basis of Section 2, we discuss the strong rooted property of $\left(E_{n}^{3}\right)$ further. We give first the following:

Lemma 3. If $\operatorname{deg} P_{1}^{*}\left(1, w_{2}, w_{3}\right) \leqq n-1$, then $I S_{1}^{\prime} \subset I S_{2} \cup I S_{3} ;$ if $\operatorname{deg} P_{1}^{*}\left(1, w_{2}, w_{3}\right)=n$, then $I S_{1}^{\prime}=\left\{\left(0, W_{2}, W_{3}, 0\right) \in C P^{3} ; \quad W_{2}, W_{3} \in C^{1}\right\}$. For $I S_{2}^{\prime}$ and $I S_{3}^{\prime}$, the conclusions hold analogously.

Proof. Consider the following two-dimensional system:

$$
\left\{\begin{array}{l}
\frac{d W_{2}}{d T^{\prime}}=W_{2} P_{1}^{*}\left(1, W_{2}, W_{3}\right)-P_{2}^{*}\left(1, W_{2}, W_{3}\right) \equiv E\left(W_{2}, W_{3}\right) \\
\frac{d W_{3}}{d T^{\prime}}=W_{3} P_{1}^{*}\left(1, W_{2}, W_{3}\right)-P_{3}^{*}\left(1, W_{2}, W_{3}\right) \equiv F\left(W_{2}, W_{3}\right) .
\end{array}\right.
$$

By the definition of $I S_{1}^{\prime}$ in Section 2,

$$
I S_{1}^{\prime}=\left\{\left(0, W_{2}, W_{3}, 0\right) ;\left(W_{2}, W_{3}, 0\right) \text { is the infinite singular point of }(3.1)\right\}
$$

Let $k=\max \left\{\operatorname{deg} E\left(W_{2}, W_{3}\right), \operatorname{deg} F\left(W_{2}, W_{3}\right)\right\}$, then $k \leqq n+1$, and

$$
\bar{E}\left(W_{2}, W_{3}, M\right)=M^{k} E\left(\frac{W_{2}}{M}, \frac{W_{3}}{M}\right)=
$$




$$
\begin{aligned}
& =W_{2} \frac{M^{n} P_{1}^{*}\left(1, \frac{W_{2}}{M}, \frac{W_{3}}{M}\right)}{M^{n-k+1}}-\frac{M^{n} P_{2}^{*}\left(1, \frac{W_{2}}{M}, \frac{W_{3}}{M}\right)}{M^{n-k}} \\
& =W_{2} \frac{P_{1}^{*}\left(M, W_{2}, W_{3}\right)}{M^{n-k+1}}-\frac{P_{2}^{*}\left(M, W_{2}, W_{3}\right)}{M^{n-k}} . \\
\bar{F}\left(W_{2}, W_{3}, M\right) & =M^{k} F\left(\frac{W_{2}}{M}, \frac{W_{3}}{M}\right) \\
& =W_{3} \frac{P_{1}^{*}\left(M, W_{2}, W_{3}\right)}{M^{n+k}}-\frac{P_{3}^{*}\left(M, W_{2}, W_{3}\right)}{M^{n-k}} .
\end{aligned}
$$

By the definition of $I S$ of $\left(E_{n}^{2}\right)$,

As

$$
I S_{1}^{\prime}=\left\{\left(0, W_{2}, W_{3}, 0\right) ;\left(W_{2}, W_{3}, 0\right) \in C P^{2} \text { and } W_{2} F^{*}\left(W_{2}, W_{3}\right)-W_{3} E^{*}\left(W_{2}, W_{3}\right)=0\right\} \text {. }
$$

then

$$
E^{*}\left(W_{2}, W_{3}\right)=\bar{E}\left(W_{2}, W_{3}, 0\right), F^{*}\left(W_{2}, W_{3}\right)=\bar{F}\left(W_{2}, W_{3}, 0\right)
$$

$$
\begin{aligned}
W_{2} F^{*}\left(W_{2}, W_{3}\right)-W_{3} E^{*}\left(W_{2}, W_{3}\right) & =\left.\left(W_{2} \bar{F}\left(W_{2}, W_{3}, M\right)-W_{3} \bar{E}\left(W_{2}, W_{3}, M\right)\right)\right|_{M=0} \\
& =\left.\left(\frac{W_{3} P_{2}^{*}\left(M, W_{2}, W_{3}\right)}{M^{n-k}}-\frac{W_{2} P_{3}^{*}\left(M, W_{2}, W_{3}\right)}{M^{n-k}}\right)\right|_{M=0} .
\end{aligned}
$$

There are three cases:

(1) If $k=n+1$, i.e. $\operatorname{deg} P_{1}^{*}\left(1, W_{2}, W_{3}\right)=n$, then

$$
I S_{1}^{\prime}=\left\{\left(0, W_{2}, W_{3}, 0\right) \in C P^{3} ; W_{2}, W_{3} \in C^{1}\right\} .
$$

(2) If $k=n$, then

$$
I S_{1}^{\prime}=\left\{\left(0, W_{2}, W_{3}, 0\right) \in C P^{3} ; W_{2} P_{3}^{*}\left(0, W_{2}, W_{3}\right)-W_{3} P_{2}^{*}\left(0, W_{2}, W_{3}\right)=0\right\}
$$

and $\operatorname{deg} P_{1}^{*}\left(1, W_{2}, W_{3}\right) \leqq n-1$. As $P_{1}^{*}\left(W_{1}, W_{2}, W_{3}\right)$ is homogeneous polynomial of degree $n$, hence $P_{1}^{*}\left(0, W_{2}, W_{3}\right) \equiv 0$.

As

and

$$
\begin{aligned}
I S_{2}= & \left\{\left(W_{1}, 1, W_{3}, 0\right) ; W_{1} P_{2}^{*}\left(W_{1}, 1, W_{3}\right)-P_{1}^{*}\left(W_{1}, 1, W_{3}\right)=0\right. \text { and } \\
& \left.W_{3} P_{2}^{*}\left(W_{1}, 1, W_{3}\right)-P_{3}^{*}\left(W_{1}, 1, W_{3}\right)=0\right\}
\end{aligned}
$$

then

$$
\begin{aligned}
I S_{3}= & \left\{\left(W_{1}, W_{2}, 1,0\right) ; W_{1} P_{3}^{*}\left(W_{1}, W_{2}, 1\right)-P_{1}^{*}\left(W_{1}, W_{2}, 1\right)=0\right. \text { and } \\
& \left.W_{2} P_{3}^{*}\left(W_{1}, W_{2}, 1\right)-P_{2}^{*}\left(W_{1}, W_{2}, 1\right)=0\right\}
\end{aligned}
$$

and

$$
I S_{1}^{\prime} \cap\left\{\left(0,1, W_{3}, 0\right) ; W_{3} \in C^{1}\right\} \subset I S_{2}
$$

$$
I S_{1}^{\prime} \cap\left\{\left(0, W_{2}, 1,0\right) ; W_{2} \in C^{1}\right\} \subset I S_{3} \text {. }
$$


Therefore $I S_{1}^{\prime} \subset I S_{2} \cup I S_{3}$.

(3) If $k \leqq n-1$, then $\operatorname{deg} P_{1}^{*}\left(1, W_{2}, W_{3}\right) \leqq n-1$, i.e.

$$
P_{1}^{*}\left(0, W_{2}, W_{3}\right) \equiv 0
$$

Hence we can assume

$$
P_{1}^{*}\left(W_{1}, W_{2}, W_{3}\right)=W_{1}\left[P_{11}^{*}\left(W_{2}, W_{3}\right)+P_{12}^{*}\left(W_{1}, W_{2}, W_{3}\right)\right]
$$

where $P_{11}^{*}\left(W_{2}, W_{3}\right)$ and $P_{12}^{*}\left(W_{1}, W_{2}, W_{3}\right)$ are all homogeneous polynomials of degree $(n-1)$ and

$$
\operatorname{deg} P_{12}^{*}\left(1, W_{2}, W_{3}\right) \leqq n-2
$$

Let

$$
\begin{aligned}
& P_{2}^{*}\left(W_{1}, W_{2}, W_{3}\right)=P_{21}^{*}\left(W_{2}, W_{3}\right)+P_{22}^{*}\left(W_{1}, W_{2}, W_{3}\right) \\
& P_{3}^{*}\left(W_{1}, W_{2}, W_{3}\right)=P_{31}^{*}\left(W_{2}, W_{3}\right)+P_{32}^{*}\left(W_{1}, W_{2}, W_{3}\right)
\end{aligned}
$$

where $P_{i 1}^{*}\left(W_{2}, W_{3}\right)$ and $P_{i 2}^{*}\left(W_{1}, W_{2}, W_{3}\right)$ are all homogeneous polynomials of degree $n$ and

$$
\operatorname{deg} P_{i 2}^{*}\left(1, W_{2}, W_{3}\right) \leqq n-1
$$

which imply $P_{i 2}^{*}\left(0, W_{2}, W_{3}\right) \equiv 0(i=2,3)$. As

therefore

$$
k=\max \left(\operatorname{deg} E\left(W_{2}, W_{3}\right), \operatorname{deg} F\left(W_{2}, W_{3}\right)\right) \leqq n-1,
$$

Hence

$$
\begin{aligned}
& W_{2} P_{11}^{*}\left(W_{2}, W_{3}\right)-P_{21}^{*}\left(W_{2}, W_{3}\right) \equiv 0 \\
& W_{3} P_{11}^{*}\left(W_{2}, W_{3}\right)-P_{31}^{*}\left(W_{2}, W_{3}\right) \equiv 0
\end{aligned}
$$

$$
W_{3} P_{21}^{*}\left(W_{2}, W_{3}\right) \equiv W_{2} P_{31}^{*}\left(W_{2}, W_{3}\right)
$$

Therefore

$$
W_{3} P_{2}^{*}\left(W_{1}, W_{2}, W_{3}\right)-W_{2} P_{3}^{*}\left(W_{1}, W_{2}, W_{3}\right) \equiv W_{3} P_{22}^{*}\left(W_{1}, W_{2}, W_{3}\right)-W_{2} P_{32}^{*}\left(W_{1}, W_{2}, W_{3}\right)
$$

So, by the definition of $I S_{2}$ and $I S_{3}$,

Therefore

$$
\begin{aligned}
I S_{2}= & \left\{\left(W_{1}, 1, W_{3}, 0\right) ; W_{1} P_{2}^{*}\left(W_{1}, 1, W_{3}\right)-P_{1}^{*}\left(W_{1}, 1, W_{3}\right)=0\right. \text { and } \\
& \left.W_{3} P_{22}^{*}\left(W_{1}, 1, W_{3}\right)-P_{32}^{*}\left(W_{1}, 1, W_{3}\right)=0\right\}, \\
I S_{3}= & \left\{\left(W_{1}, W_{2}, 1,0\right) ; W_{1} P_{3}^{*}\left(W_{1}, W_{2}, 1\right)-P_{1}^{*}\left(W_{1}, W_{2}, 1\right)=0\right. \text { and } \\
& \left.W_{2} P_{32}^{*}\left(W_{1}, W_{2}, 1\right)-P_{22}^{*}\left(W_{1}, W_{2}, 1\right)=0\right\} .
\end{aligned}
$$

$$
\left\{\left(0,1, W_{3}, 0\right) ; W_{3} \in C^{1}\right\} \subset I S_{2},
$$


and

Hence

$$
\left\{\left(0, W_{2}, 1,0\right) ; W_{2} \in C^{1}\right\} \subset I S_{3}
$$

$$
\left\{\left(0, W_{2}, W_{3}, 0\right) \in C P^{3} ; W_{2}, W_{3} \in C^{1}\right\} \subset I S_{2} \cup I S_{3} \text {. }
$$

In particular, $I S_{1}^{\prime} \subset I S_{2} \cup I S_{3}$.

From (1), (2) and (3), the conclusion of Lemma 3 holds.

Theorem 3. If $\left(E_{n}^{3}\right)$ satisfies one of the following three inequalities:

$$
\begin{aligned}
& \operatorname{deg} P_{1}^{*}\left(1, w_{2}, w_{3}\right) \leqq n-1, \\
& \operatorname{deg} P_{2}^{*}\left(w_{1}, 1, w_{3}\right) \leqq n-1, \\
& \operatorname{deg} P_{3}^{*}\left(w_{1}, w_{2}, 1\right) \leqq n-1,
\end{aligned}
$$

then for any given point $W_{0} \in C^{3}$ which is not a finite singular point of $\left(E_{n}^{3}\right)$, the phase surface $\Sigma\left(W_{0}\right)$ has some singular point at infinity as its limit point.

Proof. Without loss of generality, we assume $\operatorname{deg} P_{3}^{*}\left(w_{1}, w_{2}, 1\right) \leqq n-1$, then $P_{3}^{*}\left(w_{1}, w_{2}, 0\right) \equiv 0$, and, by Lemma 3 above, $I S_{3}^{\prime} \subset I S_{1} \cup I S_{2}$.

By Theorem 1 in Section $1, \Sigma=\Sigma\left(W_{0}\right)$ has some point at infinity $\left(W_{1}^{0}, W_{2}^{0}, W_{3}^{0}, 0\right) \in C P^{3}$ as its limit point. There are two cases:

(I) If $W_{3}^{0} \neq 0$, by Lemma 2 in Section $2, \Sigma$ must have some point at infinity in $I S_{3} \cup I S_{3}^{\prime}$ as its limit point. As $I S_{3}^{\prime} \subset I S_{1} \cup I S_{2}$, then the conclusion holds.

(II) If $W_{3}^{0}=0$, without loss of generality, assume $W_{1}^{0} \neq 0$, again by Lemma $2, \Sigma$ must have some point at infinity in $I S_{1} \cup I S_{1}^{\prime}$ as its limit point. If $\Sigma$ has limit point in $I S_{1}$, then the conclusion holds. If $\Sigma$ has limit point in $I S_{1}^{\prime}$, saying $\left(0, \bar{W}_{2}^{0}, \bar{W}_{3}^{0}, 0\right)$, there are two subcases:

(1) If $\bar{W}_{3}^{0} \neq 0$, by (I), the conclusion holds.

(2) If $\bar{W}_{3}^{0}=0$, then $\bar{W}_{2}^{0} \neq 0$, i.e. $\Sigma$ has $(0,1,0,0) \in C P^{3}$ as its limit point. Take the transformation $T_{2}$ :

$$
w_{1}=\frac{W_{1}}{M}, w_{2}=\frac{1}{M}, w_{3}=\frac{W_{3}}{M}
$$

then $\left(E_{n}^{3}\right)$ is transformed into

$$
\left\{\begin{array}{l}
\frac{d W_{1}}{d T^{\prime}}=W_{1} \bar{P}_{2}\left(W_{1}, 1, W_{3}, M\right)-\bar{P}_{1}\left(W_{1}, 1, W_{3}, M\right) \\
\frac{d W_{3}}{d T^{\prime}}=W_{3} \bar{P}_{2}\left(W_{1}, 1, W_{3}, M\right)-\bar{P}_{3}\left(W_{1}, 1, W_{3}, M\right) \\
\frac{d M}{d T^{\prime}}=M \bar{P}_{2}\left(W_{1}, 1, W_{3}, M\right)
\end{array}\right.
$$


and $\Sigma$ corresponds to $\Sigma_{2}$. As $\Sigma$ has $(0,1,0,0) \in C P^{3}$ as its limit point, $(0,0,0) \in \bar{\Sigma}_{2}$. There are still two cases:

(i) If $(0,0,0)$ is a finite singular point of (3.2), then, by the definition of $I S_{2}$, $(0,1,0,0) \in I S_{2}$, and the conclusion holds.

(ii) If $(0,0,0)$ is not any finite singular point of (3.2), let $\Sigma_{0}$ be the phase surface of (3.2) through $(0,0,0) \in C^{3}$, as $P_{3}^{*}\left(w_{1}, w_{2}, 0\right) \equiv 0$, then

$$
\Sigma_{0}=\left\{\left(W_{1}, 0,0\right) ; W_{1} \in \Sigma_{0}^{\prime}\right\}
$$

where $\Sigma_{0}^{\prime}$ is the phase surface through $W_{1}=0$ of the following one-dimensional equation

$$
\frac{d W_{1}}{d T^{\prime}}=W_{1} P_{2}^{*}\left(W_{1}, 1,0\right)-P_{1}^{*}\left(W_{1}, 1,0\right)
$$

and

$$
\left.\left(W_{1} P_{2}^{*}\left(W_{1}, 1,0\right)-P_{1}^{*}\left(W_{1}, 1,0\right)\right)\right|_{W_{1}=0}=P_{1}^{*}(0,1,0) \neq 0
$$

By Proposition 2 in Section $1, \Sigma_{0}^{\prime}=C^{1} \backslash C_{0}$ where $C_{0}=\left\{W_{1} \in C^{1} ; W_{1} P_{2}^{*}\left(W_{1}, 1,0\right)-\right.$ $\left.P^{*}\left(W_{1}, 1,0\right)=0\right\}$, and hence $\bar{\Sigma}_{0}^{\prime}=C^{1}$. Since $(0,0,0) \in \bar{\Sigma}_{2}$, by Proposition 1 in Section 1 ,

$$
\bar{\Sigma}_{2} \supset \bar{\Sigma}_{0}=\left\{\left(W_{1}, 0,0\right) ; W_{1} \in C^{1}\right\}
$$

Therefore for any $W_{1} \in C^{1}, \Sigma$ has the point at infinity $\left(W_{1}, 1,0,0\right) \in C P^{3}$ as its limit point.

On the other hand, let $\Sigma_{0}^{\prime \prime}$ be the phase surface through $(0,0)$ of the following two-dimensional equation

$$
\begin{aligned}
& \frac{d W_{1}}{d T^{\prime}}=W_{1} P_{2}^{*}\left(W_{1}, 1, W_{3}\right)-P_{1}^{*}\left(W_{1}, 1, W_{3}\right) \\
& \frac{d W_{3}}{d T^{\prime}}=W_{3} P_{2}^{*}\left(W_{1}, 1, W_{3}\right)-P_{3}^{*}\left(W_{1}, 1, W_{3}\right) .
\end{aligned}
$$

Then

$$
\Sigma_{0}=\left\{\left(W_{1}, W_{3}, 0\right) ;\left(W_{1}, W_{3}\right) \in \Sigma_{0}^{\prime \prime}\right\}
$$

As $\Sigma_{0}^{\prime \prime}=\left\{\left(W_{1}, 0\right) ; W_{1} \in \Sigma_{0}^{\prime}\right\}, \Sigma_{0}^{\prime \prime}$ has the point at infinity $(1,0,0) \in C P^{2}$ as its limit point. With the similar argument to the last part of proof of Theorem 2 in Section $2, \Sigma$ has the point at infinity $(1,0,0,0) \in C P^{3}$ as its limit point.

So $\Sigma$ has all points in $\left\{\left(W_{1}, W_{2}, 0,0\right) \in C P^{3} W_{1}, W_{2} \in C^{1}\right\}$ as its limit points. Especially, as $I S_{3}^{\prime} \neq \varnothing, \Sigma$ has all points in $I S_{3}^{\prime}$ as its limit points. Since $I S_{3}^{\prime} \subset I S_{1} \cup I S_{2}$, the conclusion holds.

This completes our proof.

Example 4. For the three-dimensional system 


$$
\left\{\begin{array}{l}
\frac{d w_{1}}{d T}=-w_{2} \\
\frac{d w_{2}}{d T}=w_{1} \\
\frac{d w_{3}}{d T}=0
\end{array}\right.
$$

$n=1, P_{1}^{*}\left(w_{1}, w_{2}, w_{3}\right)=-w_{2}, P_{2}^{*}\left(w_{1}, w_{2}, w_{3}\right)=w_{1}, P_{3}^{*}\left(w_{1}, w_{2}, w_{3}\right)=0$. As $\operatorname{deg} P_{3}^{*}\left(w_{1}, w_{2}, 1\right)=$ $0<1$, by Theorem 3 above, any phase surface which is not through $(0,0,0) \in C^{3}$ has some singular point at infinity in $I S_{1} \cup I S_{2} \cup I S_{3}$ as its limit point. In fact, by concrete calculation,

$$
\begin{gathered}
I S_{1}=I S_{2}=\{(1, \pm i, 0,0)\} \\
I S_{3}=\{(0,0,1,0)\}
\end{gathered}
$$

This system has general phase surface

Let

$$
\begin{gathered}
\Sigma: w_{1}^{2}+w_{2}^{2}=c_{1} \\
w_{3}=c_{2} .
\end{gathered}
$$

$$
w_{1}=\frac{W_{1}}{M}, w_{2}=\frac{W_{2}}{M}, w_{3}=\frac{W_{3}}{M}
$$

$\Sigma$ is extended into $\Sigma^{\prime}$ in $C P^{3}$

$$
\begin{gathered}
\Sigma^{\prime}: W_{1}^{2}+W_{2}^{2}-c_{1} M^{2}=0 \\
W_{3}-c_{2} M=0 .
\end{gathered}
$$

It is easy to verify that $\Sigma$ has two singular points at infinity in $I S_{1}=I S_{2}$ as its limit points.

In the real domain, there is no parallel result for the system in Example 4. This is because any solution curve of it is bounded.

\section{Some remarks}

According to Y. S. Chin's new approach to Hilbert's 16th problem $[2,3]$, for the 2-dimensional real polynomial system

$$
\left\{\begin{array}{l}
\frac{d u}{d t}=P_{n}(u, x) \\
\frac{d x}{d t}=Q_{n}(u, x)
\end{array}\right.
$$


let

$$
w=u+i v \quad z=x+i y \quad T=t+i \tau(i=\sqrt{-1})
$$

Then $\left(E_{n}\right)$ is extended naturally into the complex polynomial system

$$
\left\{\begin{array}{l}
\frac{d w}{d T}=P_{n}(w, z) \\
\frac{d z}{d T}=Q_{n}(w, z) .
\end{array}\right.
$$

By investigating the structure of the totality of the set of all solution surfaces of $\left(E_{n}^{*}\right)$, we can deduce conclusions for $\left(E_{n}\right)$.

If $\left(E_{n}^{*}\right)$ is given, the finite singular points and singular points at infinity of it are fixed, and the number of them is finite generally. We can investigate the topological properties of the singular points first, then to obtain the topological properties of the solution surfaces attached with the singular points, and hence, by Strong Rooted Theorem of $\left(E_{n}^{2}\right)$, to deduce some important results for the family of solution curves of the corresponding real system $\left(E_{n}\right)$.

As an application of Strong Rooted Theorem of $\left(E_{n}^{2}\right)$ in Section 2, we have discussed the existence of global general solutions of complex polynomial systems and obtained their representation theorem for complex normal polynomial systems (see X. Q. Zhao and Y. S. Chin, Science in China (Series A), 36 (1993), 394-407).

Acknowledgement. The author is very grateful to Prof. Yuan-xun Qin (Y. S. Chin) for his guidance and Prof. Ke-ying Guan of Beijing University of Aeronautics and Astronautics for useful discussions .

\section{REFERENCES}

1. L. V. Ahlfors, Complex Analysis, Third Edition (McGraw-Hill Book Co., 1979).

2. YUAN-XUN QIN, On Integral Surfaces Defined by Ordinary Differential Equations (in Chinese) (Northwest University Press, Xian, China, 1985).

3. Yuan-xun QIn (Y. S. Chin), On Surfaces Defined by Ordinary Differential Equations, $A$ New Approach to Hilbert's 16th Problem, Ordinary and Partial Differential Equations (B. D. Sleeman and R. J. Jarvis, eds.), Springer-Verlag, Lecture Notes in Math. 1151 (1985), 115-131.

4. YuAn-xun QIN, New Progress in the Theory of Surfaces Defined by Ordinary Differential Equations, Kexue Tongbao 33 (1988), 1361-1363.

5. Xiao-Qiang Zhao, On Basic Theorems in The Complex Qualitative Theory of Ordinary Differential Equations, Kexue Tongbao 35 (1990).

INSTITUTE OF Applied Mathematics

ACADEMIa Sinica

P.O. Box 2734

BEUING 100080

P.R. ChINA 